\title{
Improvements to longitudinal Clean Development Mechanism sampling designs for lighting retrofit projects
}

\author{
Herman Carstens*, Xiaohua Xia, Xianming Ye \\ Department of Electrical, Electronic and Computer Engineering, University of Pretoria 0002, South Africa
}

\begin{abstract}
An improved model for reducing the cost of long-term monitoring in Clean Development Mechanism (CDM) lighting retrofit projects is proposed. Cost-effective longitudinal sampling designs use the minimum numbers of meters required to report yearly savings at the $90 \%$ confidence and $10 \%$ relative precision level for duration of the project (up to 10 years) as stipulated by the CDM. Improvements to the existing model include a new non-linear Compact Fluorescent Lamp population decay model based on the Polish Efficient Lighting Project, and a cumulative sampling function modified to weight samples exponentially by recency. An economic model altering the cost function to a net present value calculation is also incorporated. The search space for such sampling models is investigated and found to be discontinuous and stepped, requiring a heuristic for optimisation; in this case the Genetic Algorithm was used. Assuming an exponential smoothing rate of 0.25 , an inflation rate of $6.44 \%$, and an interest rate of $10 \%$, results show that sampling should be more evenly distributed over the study duration than is currently considered optimal, and that the proposed improvements in model accuracy increase monitoring costs by $21.4 \%$ in present value terms.
\end{abstract}

Keywords: CDM, longitudinal sampling, Compact Fluorescent Lamp, Measurement and Verification, Polish Efficient Lighting Project

\section{Nomenclature}

$\begin{array}{ll}\text { Symbols } & \\ \text { A } & \text { population difference equation matrix } \\ B & \text { number of backup meters } \\ E & \text { daily energy use } \\ \bar{X} & \text { sample mean; } \\ & \text { in this study a random variable in distribution } \\ & \mathrm{N}\left(\mu_{i}, \sigma_{i}^{2} / n_{i}\right) \\ P & \text { cumulative relative precision } \\ H & \text { average annual lamp operating hours } \\ I & \text { last reporting year } \\ L & \text { lamp rated lifetime } \\ N & \text { lamp population size } \\ Y & \text { percentage of lamps left at rated lifetime } \\ Z & \text { Standard score of cumulative confidence } \\ a & \text { meter purchasing cost per unit } \\ \text { b } & \text { population difference equation vector } \\ b & \text { meter installation cost per unit }\end{array}$

*Corresponding author. Tel. +27 (0)12 420 6623; Fax 0867317626

Email address: herman.carstens@up.ac.za (Herman Carstens ) 


$\begin{array}{ll}c & \text { meter maintenance cost per unit per month } \\ d & \text { Consumer price index (inflation rate) } \\ n & \text { number of observations after } \\ & \text { finite population adjustment } \\ n^{\prime} & \text { number of effective observations } \\ n_{0} & \text { number of observations before population adjustment } \\ p & \text { precision relative to the mean } \\ r & \text { Minimum Attractive Rate of Return, } \\ & \text { or investment interest rate } \\ u & \text { control input } \\ \mathbf{x} & \text { population difference equation parameter vector } \\ \bar{x} & \text { sample mean } \\ z & \text { standard score of normal distribution } \\ \Gamma & \text { true cumulative standard deviation on energy use } \\ \Phi & \text { surviving proportion of lamp population } \\ \bar{\chi} & \text { cumulative sample mean } \\ \alpha & \text { population decay rated lifetime parameter } \\ \beta & \text { population decay slope parameter } \\ \gamma & \text { population decay initial value parameter } \\ \phi & \text { PELP study data } \\ \epsilon & \text { exponential decay factor } \\ \eta & \text { project inception cost } \\ \theta & \text { true cumulative mean energy use } \\ \mu & \text { true mean energy use } \\ \rho & \text { in a given year } \\ \sigma & \text { lag 1 autocorrelation coefficient } \\ \omega & \text { true standard deviation on energy use in a given year } \\ & \text { project operational cost }\end{array}$

\begin{tabular}{|c|c|}
\hline \multicolumn{2}{|l|}{ Abbreviations } \\
\hline ASHRAE & $\begin{array}{l}\text { American Society for Heating, Refrigeratio } \\
\text { and Air-conditioning Engineers }\end{array}$ \\
\hline AMS & Approved Methodology for Small scale \\
\hline CDM & Clean Development Mechanism \\
\hline CFL & Compact Fluorescent Lamp \\
\hline CL & Confidence Limit \\
\hline CPI & Consumer Price Inflation \\
\hline $\mathrm{CV}$ & Coefficient of Variance \\
\hline IPMVP & $\begin{array}{l}\text { International Performance Measurement } \\
\text { and Verification Protocol }\end{array}$ \\
\hline GA & Genetic Algorithm \\
\hline $\mathrm{M} \& \mathrm{~V}$ & Measurement and Verification \\
\hline MSE & Mean Squared Error \\
\hline PELP & Polish Efficient Lighting Project \\
\hline $\mathrm{R}$ & South African Rand \\
\hline TolCon & Tolerance on Constraints \\
\hline TolFun & Tolerance on Function values \\
\hline UNFCCC & $\begin{array}{l}\text { United Nations Framework Convention } \\
\text { for Climate Change }\end{array}$ \\
\hline $\mathrm{V}$ & Volts \\
\hline $\mathrm{W}$ & Watts \\
\hline
\end{tabular}




$\begin{array}{ll}\text { Subscripts } & \\ \text { B } & \text { baseline } \\ \mathrm{J} & \text { Number of groups } \\ \mathrm{j} & \text { group counter } \\ \mathrm{k} & \text { year counter } \\ \mathrm{t} & \text { time instant in years } \\ 0 & \text { year } 0\end{array}$

\section{Measurement and Verification in a South African Context}

South Africa's national electricity utility, Eskom, oversees more than 700 Energy Efficiency (EE) and Demand Side Management (DSM) projects, and also supplies more than $95 \%$ of the electricity used in the country - which is over $45 \%$ of that used on the continent [1]. In order to ensure project sustainability, the savings realised by these projects need to be measured and verified by an independent accredited Measurement and Verification (M\&V) body for a number of years [2].

This study focuses on monitoring plans for residential lighting projects where old incandescent lamps are replaced with energy saving Compact Fluorescent Lamps (CFLs). M\&V Engineers require two kinds of data to calculate energy usage in such projects: population survival data, and daily energy use data [3]. Energy usage data are obtained from electricity meters installed in a statistically representative number of lamps, whilst population survival data is collected through surveys. For this study, population survival data is assumed to be known and thus no sampling design will be devised for this component of energy use, although a population decay model will be proposed.

International guidelines [4] suggest that $M \& V$ costs should not exceed $10 \%$ of savings, but the time horizons on these projects span many years; for these projects to be eligible under the United Nations Framework Convention for Climate Change (UNFCCC) Clean Development Mechanism (CDM), the energy saving performance of lighting projects should be tracked for up to 10 years, whilst other projects may be tracked for up to 21 years. Certain stringent statistical requirements on measured data also apply: for projects to be eligible for recognition under CDM guidelines, key parameters for calculating savings are to be reported at a statistical confidence level of $90 \%$, and a relative precision around the mean of $10 \%$, known as the $90 / 10$ criterion. Although other leading guidelines recommend an 80/20 level [5], [6], [7], the 90/10 criterion will be used for this study. Due to the long planning horizons, nonoptimal metering may therefore affect savings detrimentally, or may report savings with inadequate confidence and precision. As such, cost effective longitudinal sampling designs for energy efficiency projects should form an integral part of long-term M\&V plans.

Lighting projects are chosen because these are relatively simple to model. Projects involve large populations adequately described by simple statistics and binomial working or failed states.

Research grounding the theory of $\mathrm{M} \& \mathrm{~V}$ is underway [8], but there is a need to establish best practice by the application of statistics to the specific challenges in energy monitoring and performance evaluation. This paper proposes certain improvements on current models for longitudinal meter sampling designs to ensure cost effective and accurate performance tracking.

\section{Performance Tracking in Literature}

\subsection{General Performance Tracking Literature}

Very little literature pertaining to this specific problem exists. The International Performance Measurement and Verification Protocol (IPMVP) [4], for example, does advise that the 90/10 criterion be used, but does not provide specific guidance regarding implementation.

Both the IPMVP and The American Society for Heating, Refrigeration and Air Conditioning Engineers' (ASHRAE) Guideline on Measurement of Energy and Demand Savings [6] make a useful distinction between the different kinds of uncertainty encountered during an $M \& V$ study. Measurement uncertainty occurs due to equipment inaccuracy: incorrect selection, calibration, installation or operation. Modelling uncertainty arises from inappropriate mathematical models being used: not considering all covariates, for example. Sampling uncertainty pertains to quantifiable uncertainties arising from not measuring the whole population. This study will focus on managing the latter kind of uncertainty in the context of project cost. 
The ASHRAE guideline is a comprehensive technical resource. This guideline uses the fractional savings approach [9] and warrants further investigation for application to $\mathrm{M} \& \mathrm{~V}$. However, it cannot be used for the problem at hand since the CDM guideline specifies that the standard confidence/precision approach is to be followed.

The UNFCCC AM0046 Guideline [10] for monitoring EE lamp retrofit projects does provide a statistical sampling framework, but it has been observed that this framework is not practical, and adoption in industry has thus been poor [11]. Moreover, the framework aims at a sound sampling and recording protocol, rather than a treatment of the statistical computation methods.

Recently, more focused studies of the problem have been conducted, specifically regarding the replacement of incandescent lamps with Compact Fluorescent Lamps (CFLs). The most notable pertains to two-sample meter cost minimisation models, where a CFL and a Light Emitting Diode (LED) group are combined in a stratified random sample weighted according to population sizes and solved using frequentist statistics [12]. This model was then applied to a case of a single population over multiple years, where population decay is also considered [13]. It was assumed that samples are independently and identically distributed (i.i.d.) because meters are placed in different households. However, this tacitly assumes that samples from different years, taken by the same meter in the same household, are independent. Such time-series data are usually autocorrelated and not independent unless it is a Gaussian 'white noise' process [14]. It has been shown that autocorrelation does exist in hourly and daily energy use data [15], but if observations have constant variance and a lag 1 autocorrelation of $\rho$ is assumed (as has been in literature [9]), then the number of effective observations $n^{\prime}$ may be modified to [16]:

$$
n^{\prime}=n \frac{1-\rho}{\rho}
$$

However, given that the nature of the autocorrelation is unknown during the modelling phase, a simple model for i.i.d. measurements may be posited as a starting point, with a recommendation that future work investigate the possibility of autocorrelation and employ the concomitant statistical tools. Therefore the two aforementioned studies will be used as a basis for this contribution, and expanded to incorporate more advanced population decay, weighting, and economic considerations.

\subsection{Literature concerning Population Models}

Let $\Phi_{t}$ express the proportion of functioning lamps surviving at time $t$, where $N_{0}$ denotes the initial population size:

$$
\Phi_{t}=\frac{N_{t}}{N_{0}}
$$

Various models for population decay have been proposed, the simplest being exponential decay [17]:

$$
\Phi_{t}=e^{-t}
$$

Such a model is not realistic however, as it implies that the product has a constant hazard rate, (denoting the failure rate for non-repairable items). This is not the case for lamps as they exhibit an ageing property where older CFLs are more likely to fail at any given point in time than newer CFLs.

The second population decay model considered is that in the AMS-II.J CDM Guideline, as implemented in current studies on which this paper is based [13]. It is a straight line graph where $H$ denotes annual operating hours and $i$ denotes years. $Y$ is the percentage of lamps left at the rated lifetime $L$ ( $Y=50$ is recommended):

$$
N_{t}= \begin{cases}N_{0}-i \times H \times \frac{100-Y}{100 \times L} & \text { for } i \times H<L \\ 0 & \text { for } i \times H \geq L\end{cases}
$$

The California Public Utilities Commission (CPUC) requires that survival analysis techniques be used to assess the effective useful life (EUL) of devices in retrofit projects [18]. These survival curves have sigmoid shapes; a phenomenon confirmed by empirical studies. For example, the Polish Efficient Lighting Project (PELP), conducted by the World Bank through the International Finance Corporation, tracked 1.2 million lamps over a number of years in order to assess various facets of such retrofit projects [19]. The results correlated with another study conducted by 


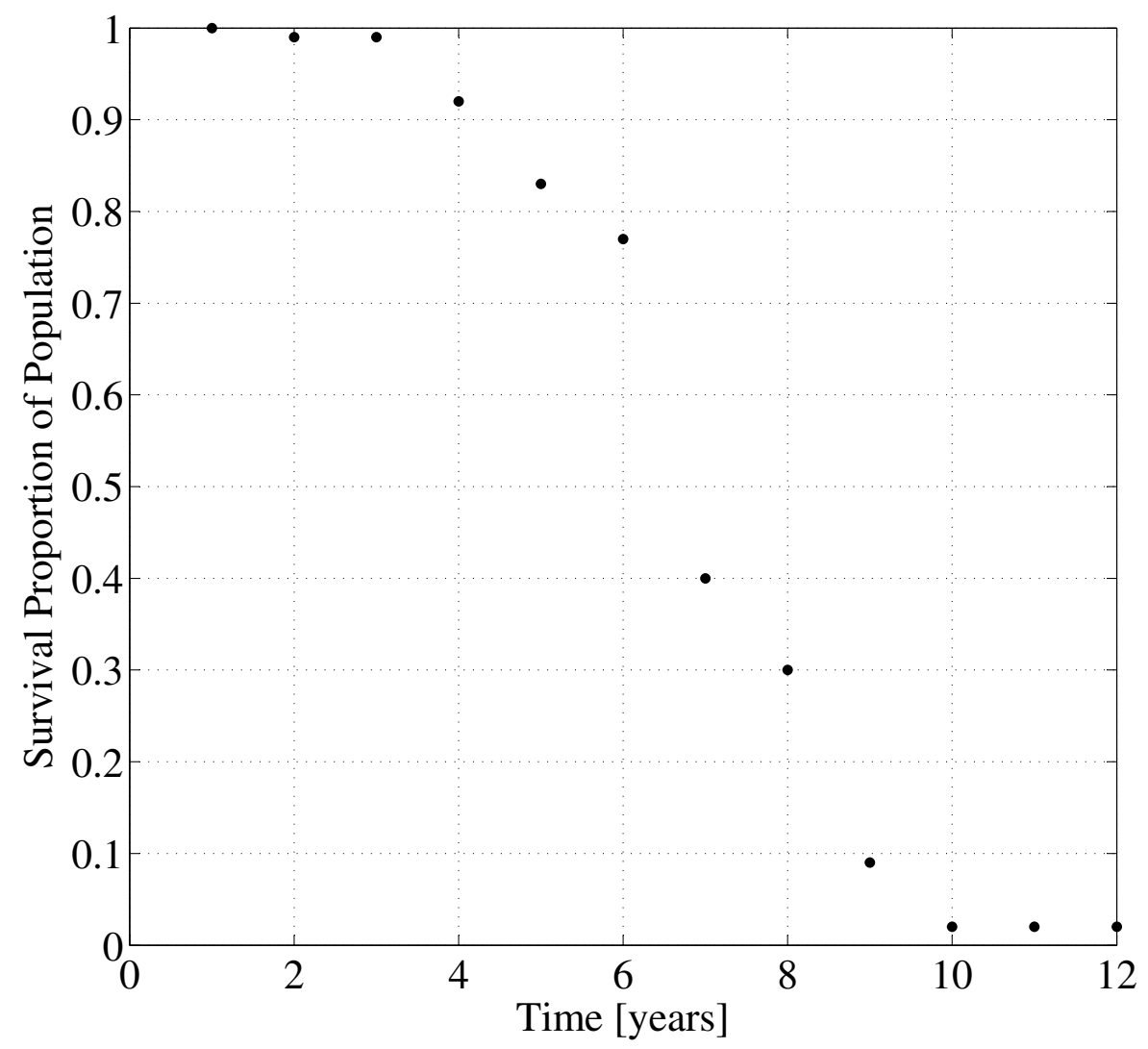

Figure 1: PELP Data [19].

the contractor in the New York area [20], and it was found that the decay rate was approximately 6.2\% p.a. The result is a logistic population decay curve shown in Figure 1.

This study is regarded as having the most reliable data for the South African context [21], and it has been proposed that the following model be fitted to these data:

$$
\Phi_{t}=\frac{1}{1+e^{t-L}} .
$$

This model is part of a family of logistic populations first proposed by Verhulst [22], and take the form

$$
\Phi_{t}=\frac{1}{1+e^{-t}} .
$$

These models were shown to describe the limiting effect that the carrying capacity of the land has on population growth, where population growth was seen as exponential and unlimited previously. It may also be used as a decay model by altering the sign of the exponent. Since the standard logistic equation is a symmetrically odd function centred around 0 , the $-L$ term was introduced in order to accommodate different lamp lives as is illustrated in Figure 2 . 


\section{Improved Population Decay Model}

\subsection{Decay model formulation}

The proposed improvement to equation (5) is similar to Lotka's reformulation of Verhulst's model as a dynamic equation with additional parameters [23]. As previous work suggests, the difference equation form of this decay model is especially applicable to the engineering context [24].

It is proposed that CFL decay be described by

$$
\frac{d \Phi}{d t}=-\beta \Phi(1-\gamma \Phi)
$$

In discrete form it is written as

$$
\Phi_{k+1}=\beta \gamma \Phi_{k}^{2} \Delta t-\beta \Phi_{k} \Delta t+\Phi_{k}
$$

and in general form:

$$
\Phi_{t}=\frac{1}{\gamma+\alpha e^{\beta t}}
$$

which could be written as:

$$
\Phi_{t}=\frac{1}{\gamma+e^{\beta t-L}}
$$

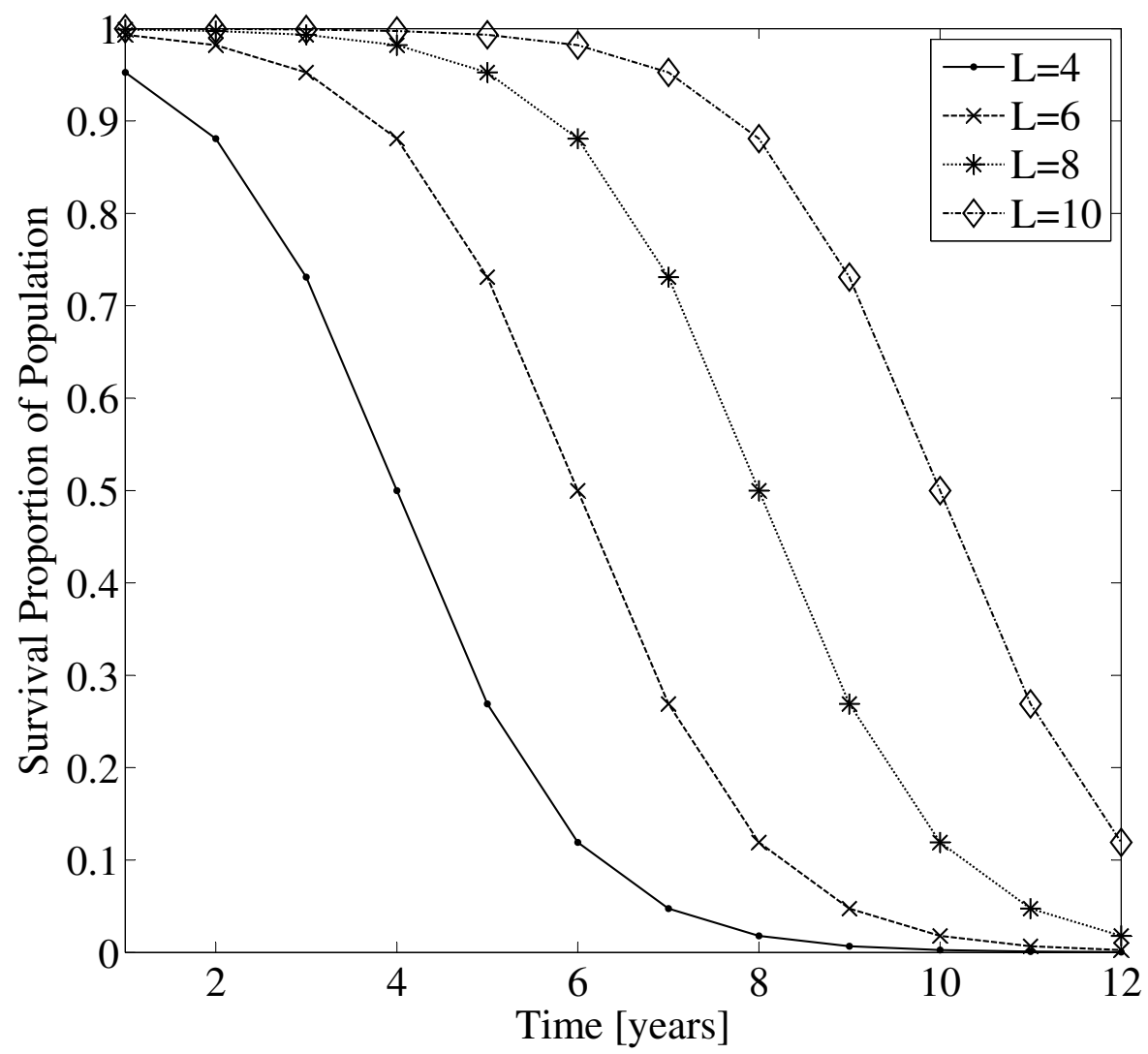

Figure 2: Decay curves from equation (5) for different lamp lives $L$. 
where:

$$
\alpha=e^{-L} \text {. }
$$

It is apparent that equation (7) is similar to equation (5) for $\beta=\gamma=1$. However, the parameter $\beta$ in equation (7) allows different decay rates to be modelled as shown in Figure 3. This is important because it is not necessarily the case that the decay rate is close to unity (even though this is shown to be the case for the PELP study [19]). With different operating conditions, different manufacturing quality control, or different technologies, it is expected that the assumption of $\beta=1$ would not necessarily hold. A steeper slope would indicate a smaller manufacturing and operating variance, with more lamps failing closer to the mean lamp life. Thus the parameter $\beta$ increases the applicability of the model significantly, and it is possible that other technologies may also be modelled in this way.

Parameter $\gamma$ is approximately inversely proportional to the starting population because at $t=0$,

$$
\Phi_{0}=\frac{1}{\gamma+e^{-L}}
$$

but for any realistic $L$,

$$
\Phi_{0} \approx \frac{1}{\gamma}=\Phi_{0}
$$

Although $\gamma=1$ should always be the case, it has been found to vary. These variations may be ascribed to project phenomena rather than true population behaviour. For example, Free Ridership (where subsidised CFLs would have been installed without the subsidy) may cause some units to be installed at a later date than project inception, since they are stored by home owners first. This would alter the initial population in a way that can be accounted for by $\gamma$. However, $\gamma$ cannot be used to account for a whole monitoring project starting after $t=0$, e.g. setting $\gamma=2$ if project monitoring starts where $N=0.5$, as the decay rate $\frac{d N}{d t}$ would approach 0 asymptotically where it should be maximum as at $t=6.8$ in Figure 4). Also, it may not be used to compensate for non-homogeneous populations, for example where only half of the population is composed of CFLs. In such a case, stratified random sampling should be used.

The difference equation formulation means that fewer data points need to be used for the identification of system parameters, since the parameter $L$ need not be determined. This also means that the lamp installation date is not relevant for determining the state of the system at $t=k+1$, as $\Phi_{k+1}$ is not a function of $t$, but only of $\Phi_{k}$ and parameters $\beta$ and $\gamma$.

The discrete form of the equation formulated in equation (8) holds three advantages [24]: First, estimated savings at $\Phi_{k+1}$ informs the project manager of the feasibility of continuing the project. Second, since survival data is binomially distributed, and for binomial data the sample size $n=f(\Phi)$, this provides information about the estimated population size (and thus required sample size) at the next time step. Third, control techniques may be applied to the problem of lamp replacement by reformulating equation (8) as

$$
\Phi_{k+1}=\beta \gamma \Phi_{k}^{2}-\beta \Phi_{k}+\Phi_{k}+u_{k}
$$

for $\Delta t=1$, where $u_{k}$ is a control input. This finds practical application in a scenario where a project developer is paid by Eskom based on the savings realised in a certain EE project. The developer would then want to optimise his control inputs (replacing lamps) over the duration of the project in order to ensure that he maximises profit.

\subsection{System Identification}

System parameters $\beta$ and $\gamma$ can be identified using a least-squares approach with decision variables $\beta$ and $\gamma$. Let $\phi_{t}$ be the surviving proportion of lamps at time $t$ in the PELP study. For year $I$ as the last reporting year, the objective function is defined as

$$
\min \frac{1}{n} \sum_{t=2}^{I}\left(\Phi_{t}-\phi_{t}\right)^{2},
$$

where $\Phi_{t}$ is defined by equation (8). The function considers data from $t=2$ and onward since $t=1$ is assumed to be known such that $\Phi_{1}=\phi_{1}$. It is found that the PELP data can be characterised by $\beta=0.921$ and $\gamma=0.986$, 


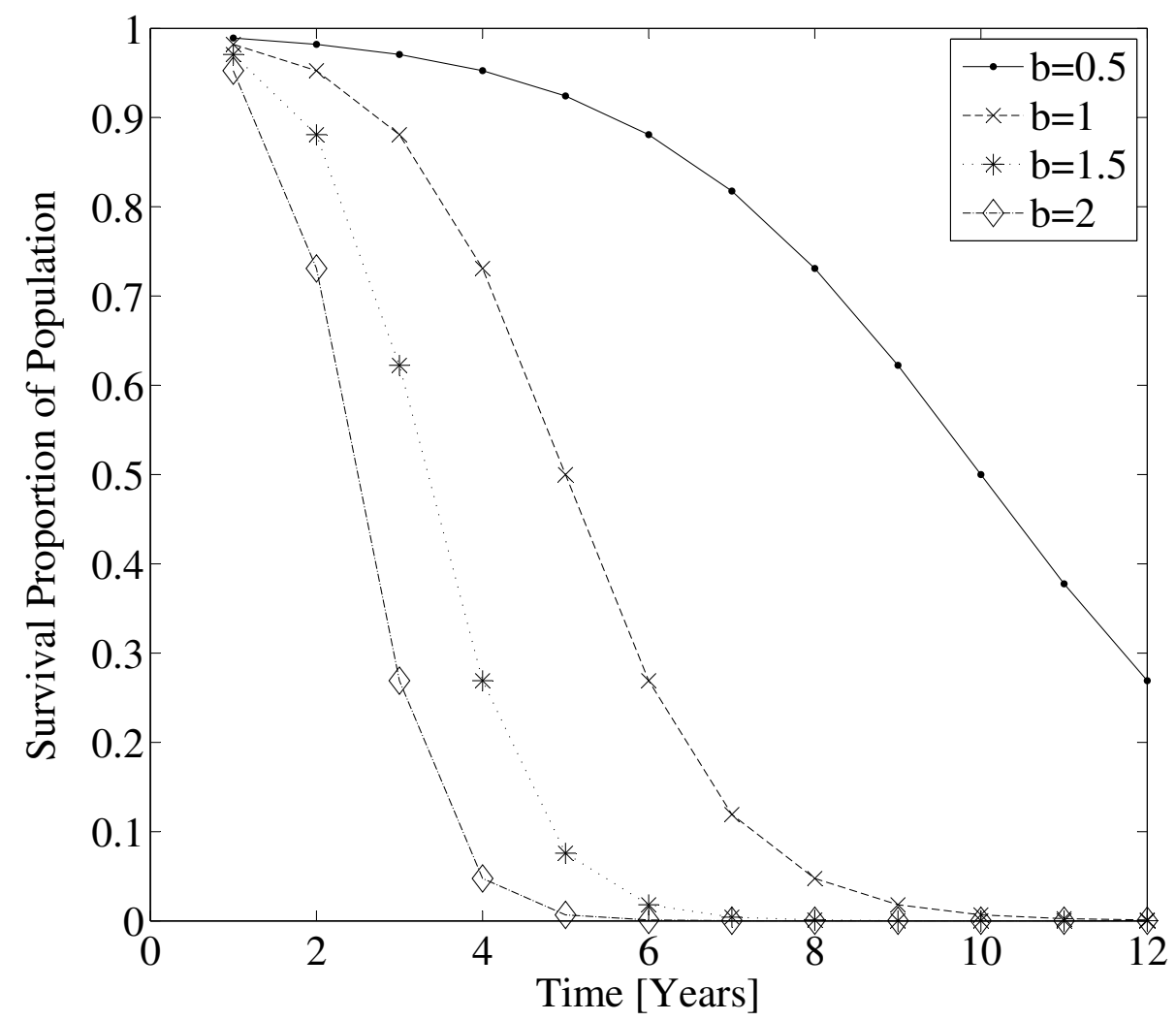

Figure 3: Decay curves for equation (10) using $L=5, c=1$, and varying $b$.

with a Mean Squared Error (MSE) of 0.0015. The result is shown in Figure 4. By way of comparison, the optimal least-squares fit of equation (5) yields $L=6.866$, with an MSE of 0.0368 .

It is noted that in practice, parameters $\beta$ and $\gamma$ may not be known accurately beforehand. Let $D_{k}$ be the data available at time $k$, in this case the sample population survival proportions. Then:

$$
\left(\Phi_{k+1} \mid D_{k}\right)=f\left(\Phi_{k}, \beta_{k}, \gamma_{k}\right),
$$

where $\beta_{k}$ and $\gamma_{k}$ are determined incrementally at each time $t=k$ so that

$$
\left(\beta_{k}, \gamma_{k}\right)=\left(\beta, \gamma \mid D_{k}\right) .
$$

Since equation (8) has two parameters instead of one, more data is needed to define the equation (8) than would be the case for equation (5). However, it is argued that a two-parameter model such as equation (8) is not merely convenient, but necessary. For example, equation (5) assumes $\beta=1$, i.e. all populations decay at the same rate (incidentally the PELP population decay rate). As such, it appears accurate for predicting the decay of the PELP data, but would not be able to predict other population survival curves satisfactorily. For this reason $\beta$ should be a variable.

The accuracy of equation (16) would be improved if $\beta$ and $\gamma$ are known or expected from previous studies. Since $\gamma \approx 1$ for all cases, it is recommended that this assumption be made in the early stages of project monitoring with unknown parameters. This reduces the number of unknown parameters to one, thereby reducing the amount of data needed to define the model accurately.

In future, population decay models may be improved by taking sample sizes into account. In the current context $D_{k}$ only contains the proportion of lamps surviving at time $t$ according to a sampling survey taken. However, $D_{k}$ 


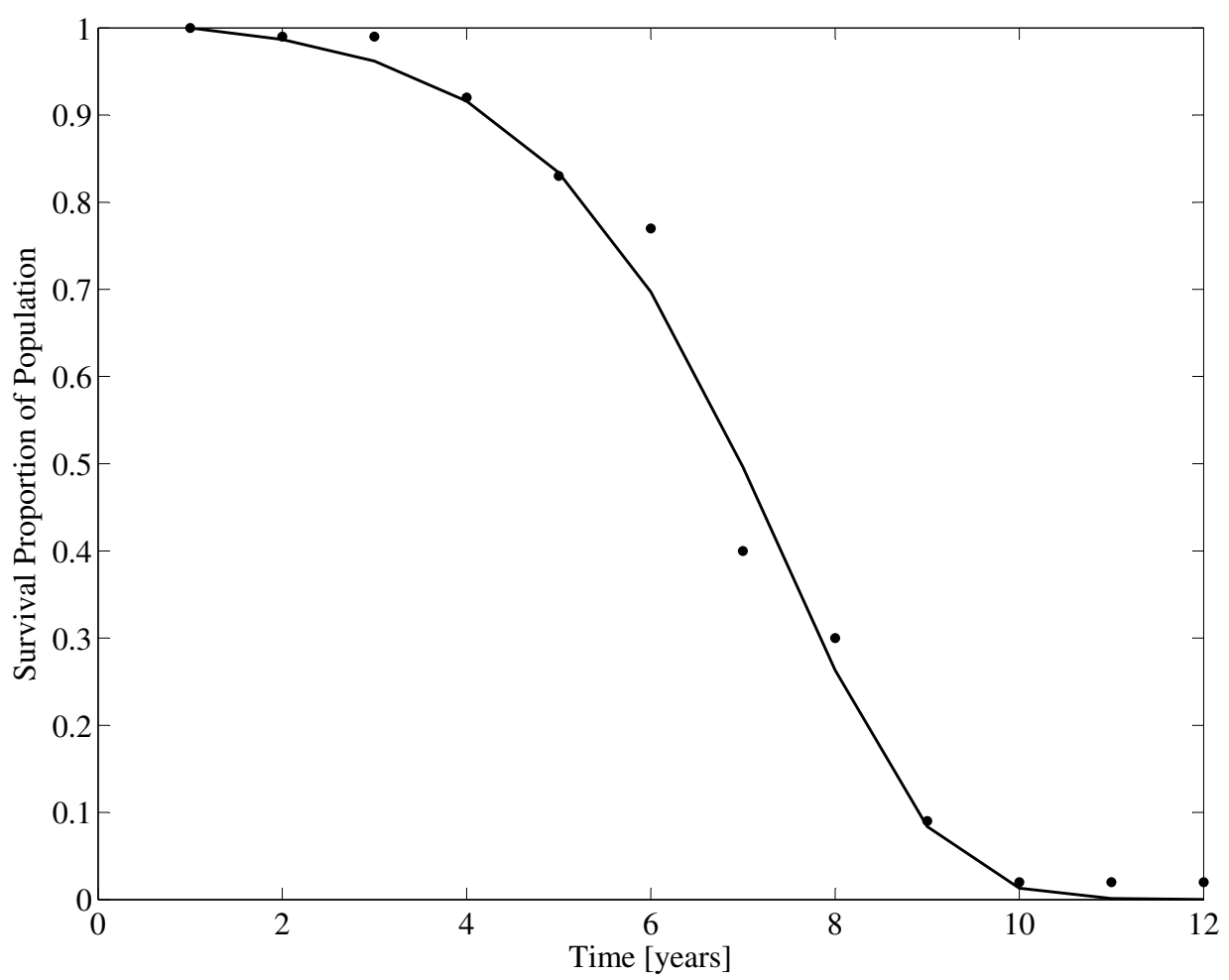

Figure 4: Equation equation (8) least-squares fit to PELP data

can also include more information such as the sizes of samples corresponding to each population proportion, as well as censoring information. Since larger samples should carry more weight when calculating model parameters than smaller samples, more accurate parameter estimation can be achieved. Such considerations can be incorporated by the use of conditional probability methods.

\section{Sampling Model Formulation}

When calculating energy savings for a lighting retrofit project with a total number of groups $J$ there are two main components that constitute energy use. The first is the average daily energy use per device $E_{j}$, and the second number of surviving devices $N_{j}$ [25]. Taking a baseline energy use per device $E_{B}$, this may then be expressed as

$$
E_{B}=\sum_{j=1}^{J}\left(N_{j} E_{j}\right) .
$$

$N_{j}$ is affected by population decay as described in Section 2.2, and $E_{j}$ is determined by metering measurements. Meter measurements will be the focus of the rest of this paper.

A statistical model for describing sequential metering samples has been described [13]. As the aforementioned model was used as the basis for the current research, the assumptions and key equations will be reproduced below.

The assumptions on which this model is based have been stated as [13]:

1. Metering data are independent and normally distributed.

2. The lamp population does not decay during the CDM-specified 90-day baseline period.

3. During the reporting period maintenance is performed on active meters only. 
There are also additional latent assumptions in the aforementioned model. It is recommended that the sensitivity of the model to these assumptions be investigated in a future study:

5. The mean energy use (the integral of the daily load profile) is stationary throughout the study.

6. Samples of the same population, taken in different years, can be treated as independent.

7. Statistical power (Type II-errors) is not considered in calculating sample size

8. The meter purchasing cost is constant in future value terms. I.e., R4,032 (South African Rand) would purchase a meter in any given year. (\$374.37, assuming an exchange rate of R10.77 to the United States Dollar)

The assumption of a stationary mean is potentially significant. First it should be noted that since the same lamps are being measured repeatedly, continuity is expected. Seasonal effects should be visible in the month-to-month energy use, however, since annual energy use is considered for calculation, they may be neglected. At a finer surveillance resolution, a model correcting for seasonality and other periodic autocorrelative effects should be implemented. Assuming a stationary mean for modelling purposes simplifies calculation, and is the preferred choice in the absence of data to the contrary. It would also be possible for account for varying parameters during actual studies, using non-routine adjustments. This is because the sampling interval allows for recalculation to be done where equation (19) could be rewritten as a less elegant summation with different mean and standard deviations for different years. Likewise, $\theta_{K}$ and $\Gamma_{K}$ below may be adjusted when this is deemed necessary.

\subsection{Calculation of mean and variance}

Because of the assumption of a stationary mean in 5 above, the cumulative sample mean $\bar{\chi}$ varies according to

$$
\bar{\chi}_{K} \sim \operatorname{Normal}\left[\theta_{K}, \Gamma_{K}^{2}\right],
$$

where the true mean $\theta_{K}$ is defined as

$$
\theta_{K}=\frac{\sum_{i=1}^{K} N_{i} \mu_{i}}{\sum_{i=1}^{K} N_{i}},
$$

and the cumulative sampling standard deviation is defined as

$$
\Gamma_{K}=\sqrt{\sum_{i=1}^{K} \frac{\sigma_{i}^{2}}{n_{i}} \cdot \frac{N_{i}^{2}}{\left(\sum_{i=1}^{K} N_{i}\right)^{2}}} .
$$

Because of the assumption of a stationary mean, samples taken at different times may be combined to give a cumulative sample size with which calculations may be done.

Assuming that a given sampling mean for year $i$ follows the distribution $\bar{X}_{i}=\mathrm{N}\left(\mu_{i}, \sigma_{i}^{2} / n_{i}\right)$, the cumulative sample mean in year $K$ is defined as

$$
\bar{\chi}_{K}=\frac{\sum_{i=1}^{K} N_{i} \bar{X}_{i}}{\sum_{i=1}^{K} N_{i}} .
$$

By substituting the variables defined above into the standard score transformation

$$
z=\frac{\bar{x}-\mu}{\sigma / \sqrt{n}}
$$

and rearranging, we find that

$$
Z_{K}=\frac{\bar{\chi}_{K}-\theta_{K}}{\Gamma_{K}}
$$

and

$$
P_{K}=\frac{\bar{\chi}_{K}-\theta_{K}}{\bar{\chi}_{K}}
$$


where $P_{K}$ and $Z_{K}$ denote the cumulative precision and standard scores up to the $K$ th crediting year.

Practically, however, the true mean $\mu$ and true standard deviation $\sigma$ are not known; only the sampling mean $\bar{x}$ and sampling standard deviation $s$ are known. In order to do simulations for meter placement planning, these values have to be assumed. By realising that if the coefficient of variation, $\mathrm{CV}$, is defined as

$$
\mathrm{CV}=\frac{\sigma}{\mu},
$$

then

$$
s=\bar{x} C V,
$$

Substituting equation (27) into equation (23), the way in which it has been formulated in previous studies [13] has been that for year $i$,

$$
Z_{i}=\frac{\sum_{j=1}^{i} \frac{N_{j} z_{j}}{\sqrt{n_{j}}}}{\sqrt{\sum_{j=1}^{i} \frac{N_{j}^{2}}{n_{j}}}}
$$

and

$$
P_{i}=\frac{\sum_{j=1}^{i} \frac{C V_{j} N_{j} z_{j}}{\sqrt{n_{j}}}}{\sum_{j=1}^{i} N_{j}} .
$$

It should be noted that this formulation does not allow for sample sizes of zero, i.e. for studies where there are years where no samples are taken. This presents a problem when optimising, as not considering sample sizes of zero constrains the problem unnecessarily. Thus, $Z$ may be formulated as

$$
Z_{i}= \begin{cases}\text { equation (28) } & \forall n_{i}>0 \\ 0 & \forall n_{i}=0\end{cases}
$$

There is no need to constrain $P_{i}$ since it is a function of $z_{i}$ for years subsequent to a zero-sample year, and it may therefore be left as undefined or 'Not a Number' $(\mathrm{NaN})$ in Matlab:

$$
P_{i}= \begin{cases}\text { equation (29) } & \forall n_{i}>0 \\ \text { undefined } & \forall n_{i}=0\end{cases}
$$

\subsection{Sample size calculation}

The well-known standard normal sampling formula shows that

$$
n_{0}=\frac{z^{2} \mathrm{CV}^{2}}{p^{2}},
$$

where $z$ is the standard score corresponding to a given confidence level.

The relative precision $p$ is defined as the maximum difference between the confidence limits (CL) and the mean, normalised with respect to the mean:

$$
p=\frac{|\mathrm{CL}-\mu|}{\mu} .
$$

Therefore, for the $90 / 10$ criterion (90\% confidence interval, $10 \%$ precision), and assuming a CV of 0.5 as is recommended in $\mathrm{M} \& \mathrm{~V}$ [4], [26] the required sample size would be

$$
n_{\text {unad justed }}=\frac{1.645^{2} \cdot 0.5^{2}}{0.1^{2}} \approx 68 .
$$

For small populations, it is necessary to include a finite population adjustment: 


$$
n_{i}=\frac{n_{\text {unadjusted }, i} N}{n_{\text {unad justed }, i}+N} .
$$

This adjustment affects the sample size for $n / N>5 \%$ [27]. By combining these equations, the required sample size is found to be

$$
n_{i}=\frac{z_{i}^{2} C V_{i}^{2} N_{i}}{z_{i}^{2} C V_{i}^{2}+N_{i} p_{i}^{2}} .
$$

\subsection{Project Cost Calculation}

Project costs may now be calculated, and consist of two parts: the project inception cost, and operational costs.

Let $a_{i}$ be the meter purchasing cost in year $i, b_{i}$ be meter installation cost, and $c_{i}$ be the monthly meter maintenance cost, all expressed in per unit Present Value. The project inception cost (which includes 3 months' maintenance in baseline period) would then be

$$
\eta=\left(a_{0}+b_{0}+3 c_{0}\right) n_{0}
$$

and project operational costs would be

$$
\omega= \begin{cases}\sum_{i=1}^{I}\left[12 c_{i} n_{i}-B_{i}\left(a_{i}+b_{i}\right)\right] & \forall B<0 \\ \sum_{i=1}^{I}\left(12 c_{i} n_{i}\right) & \forall B>0\end{cases}
$$

where $B_{i}$ is the backup meters (meters previously installed, but no longer needed) available in year $i$, defined as

$$
B_{i}=\max \left(B_{i-1}, 0\right)+n_{i-1}-n_{i}
$$

\subsection{Optimisation formulation}

Therefore one could formulate the optimisation program for a project up to year $I$ as: Decision variable:

$$
\lambda=\left(z_{1}, p_{1}, \ldots z_{I}, p_{I}\right)
$$

Objective function:

$$
\operatorname{Min} \eta+\omega
$$

Constraints:

$$
\begin{aligned}
& Z_{i} \geq 1.645 \forall i \in \delta \\
& P_{i} \leq 10 \% \forall i \in \delta .
\end{aligned}
$$

Where $\delta$ represents the set of reporting years. For example, if it is required that savings be reported in years 1,5 , and $10, \delta=(1,5,10)$.

\subsection{Model Improvement}

The model thus far is standard theory, but may be improved upon by implementing the changes discussed below.

\subsubsection{Non-linear decay model}

The first improvement that has been implemented is a non-linear population decay model as described in Section 2.2 


\subsubsection{Exponential Windowing Function}

Previously, all measurements in the time series were weighted equally as a legacy of single-time, multiple-sample models. However, in practice data obtained during earlier measurements are not accorded the same weight as data obtained more recently. Therefore an exponential decay window, akin to exponential smoothing functions used in time series analysis, has been introduced. This can be thought of as a moving weighted average.

Whereas the cumulative mean distribution was formulated as equation (20), for an exponential decay factor $\epsilon$, it is now written as

$$
\bar{\chi}_{i}=\frac{\bar{X}_{K} N_{K}}{N_{K}}+\frac{\sum_{i=1}^{K-1} \bar{X}_{i} N_{i}(1-\epsilon)^{K-i}}{\sum_{i=1}^{K-1} N_{i}(1-\epsilon)^{K-i}},
$$

thereby weighting measurements not only by sample size, but also by recency.

\subsubsection{Time-value of money considerations}

Because the kind of projects under investigation have long planning horizons, it is prudent to consider the timevalue of money when calculating project cost. Two factors were taken into account: the depreciation of meter purchasing values, and the opportunity cost incurred from spending money early in the project, when that money could have been invested to generate interest.

Let $d=6.44 \%$ be the Consumer Price Inflation (CPI) [28], and $r=10 \%$ be the Minimum Attractive Rate of Return, or investment interest rate. For year $n$, the true meter purchasing cost is calculated as

$$
a_{n}=\frac{a_{0}}{(1+d)^{n}}+\frac{a_{0}(1+r)^{K-n}}{(1+d)^{K}} .
$$

It is assumed that the meter purchasing cost stays constant in future value terms, i.e. $\mathrm{R} a_{0}$ would purchase a meter in any given year. Due to inflation, however, the meter purchasing cost declines in real terms according to the inflation rate.

Furthermore, it is assumed that the money used to purchase a meter could be invested at a rate of return of $r=10 \%$ until the end of the study in year $K$. This is the opportunity cost incurred by purchasing the meter in year $n$.

Since meter installation and maintenance costs are labour costs, it is assumed that they will increase with inflation, and thus stay constant in real value terms. However, opportunity costs are still taken into account during calculation.

\section{Case Study}

A previous case study and model [13] is used as a benchmark to ensure fair comparison. In this case study, incandescent lamps were replaced with CFLs in a number of provinces in South Africa [29]. The relevant parameters are listed in Table 2.

\subsection{Model Validation}

First, the nature of the search space was investigated. The previously reported case study was used with its reported optimal solution (labelled 'Solution 1'), and a line section was drawn to another solution ('Solution 2'), as shown in Figure 5. This proved that the search space is both stepped and discontinuous, and explains the finding that solutions given by gradient methods such as the interior-point algorithm are sensitive to the initial solution $\mathbf{x}_{0}$.

Thereafter, a cross section was drawn at a random dimension of the solution, in this case $\lambda_{7}$, and this was made to vary with $\Delta h$. It can be seen in Figure 6 that for $\lambda_{7}$ the algorithm did indeed converge on the optimal solution, and that it is constrained on one side. Although not shown here, this is also the case for the other dimensions of $\lambda$.

The cost calculation was validated by reprogramming the cost equations into Microsoft Excel and comparing the costs of various sampling plans to the results given by the Matlab subroutine.

Given the findings discussed above, it was decided that a Genetic Algorithm (GA) is appropriate for solving the problem at hand. The optimisation parameters are shown in Table 3. In order to validate the code, the model parameters were altered to $\delta=(3,8)$ and $\epsilon=0.99$, effectively constraining the model to two different sampling problems. As would be expected, the model converged on the solution of 67 meters for years 5 and 8 , and 0 meters in other years (adjusted from 68 using equation (36)). 


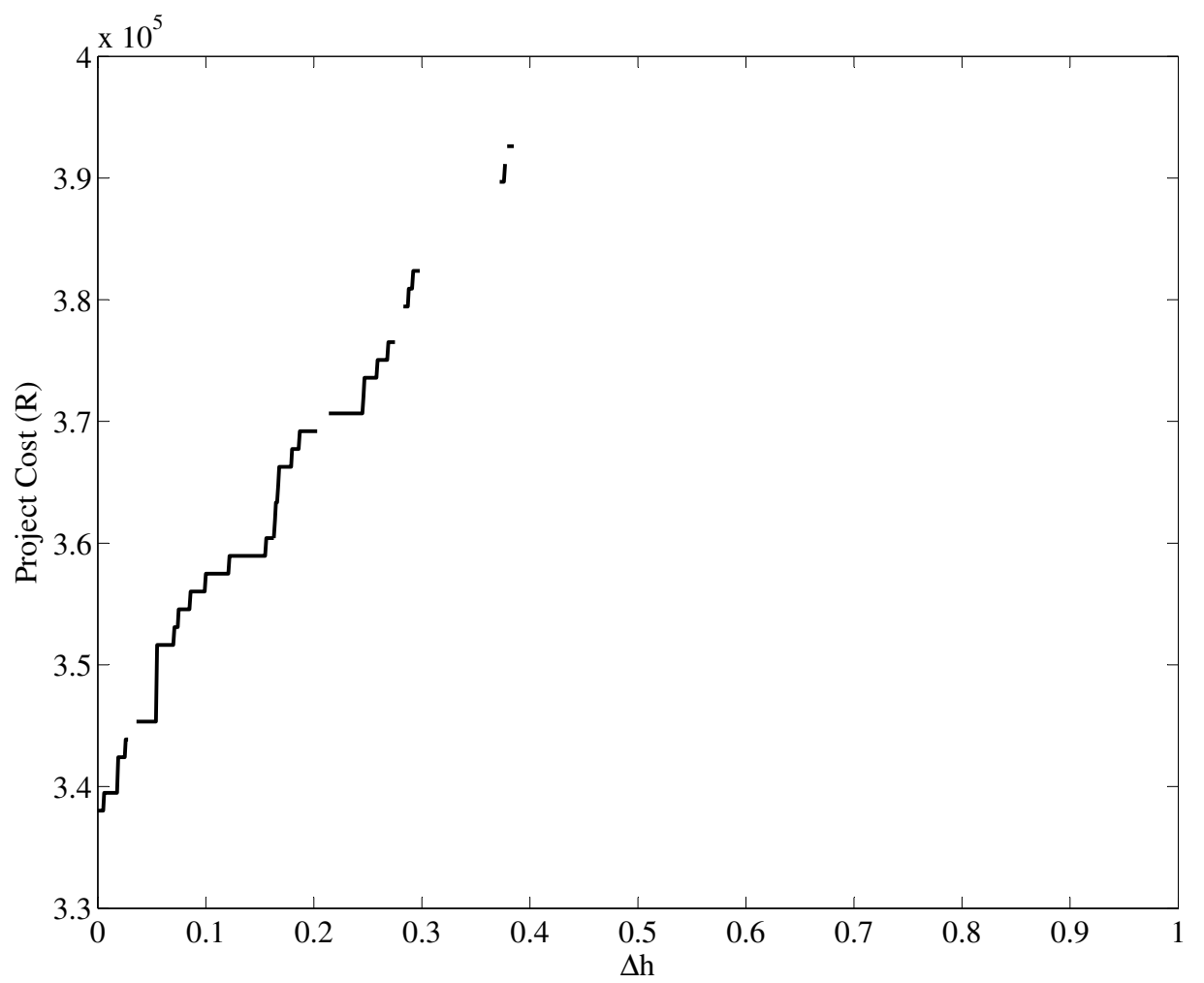

Figure 5: Line section through search space forSolution $1+h \times($ Solution $2-$ Solution 1$)$

In order to establish a basis for comparison, the original problem was first solved using the GA. Since a GA is a heuristic, it does not guarantee that a global optimum will be found. However, the optimisation parameters were set such that the heuristic converged reliably to high-quality solutions, with parameters listed in Table 3. No improved solutions to the ones originally published for this case study were found, because of the alteration made in the way the algorithm rounds decimal sample sizes. The closest the heuristic came to the original value of R338,028 $(\$ 31,386)$

Table 2: Case Study Parameter Values (before changes)

\begin{tabular}{cc}
\hline Parameter name & Value \\
\hline Reporting years & $\boldsymbol{\delta}=(2,4,6,8,10)$ \\
Meter purchasing cost & $a_{0}=\mathrm{R} 4,032$ \\
Meter installation cost & $b=\mathrm{R} 420$ \\
Meter maintenance cost & $c=\mathrm{R} 122$ \\
Coefficient of variation & $\mathrm{CV}=0.5$ \\
CPI inflation rate & $d=6.44 \%$ \\
Investment interest rate & $r=10 \%$ \\
Exponential decay factor & $\epsilon=0.25$ \\
Population size & $N_{0}=607,559$ \\
Rated lamp life & $\mathrm{L}=20,000$ hours \\
Daily usage & 4.5 hours \\
Incandescent Lamp power rating & $100 \mathrm{~W}$ \\
CFL power rating & $20 \mathrm{~W}$ \\
\hline
\end{tabular}




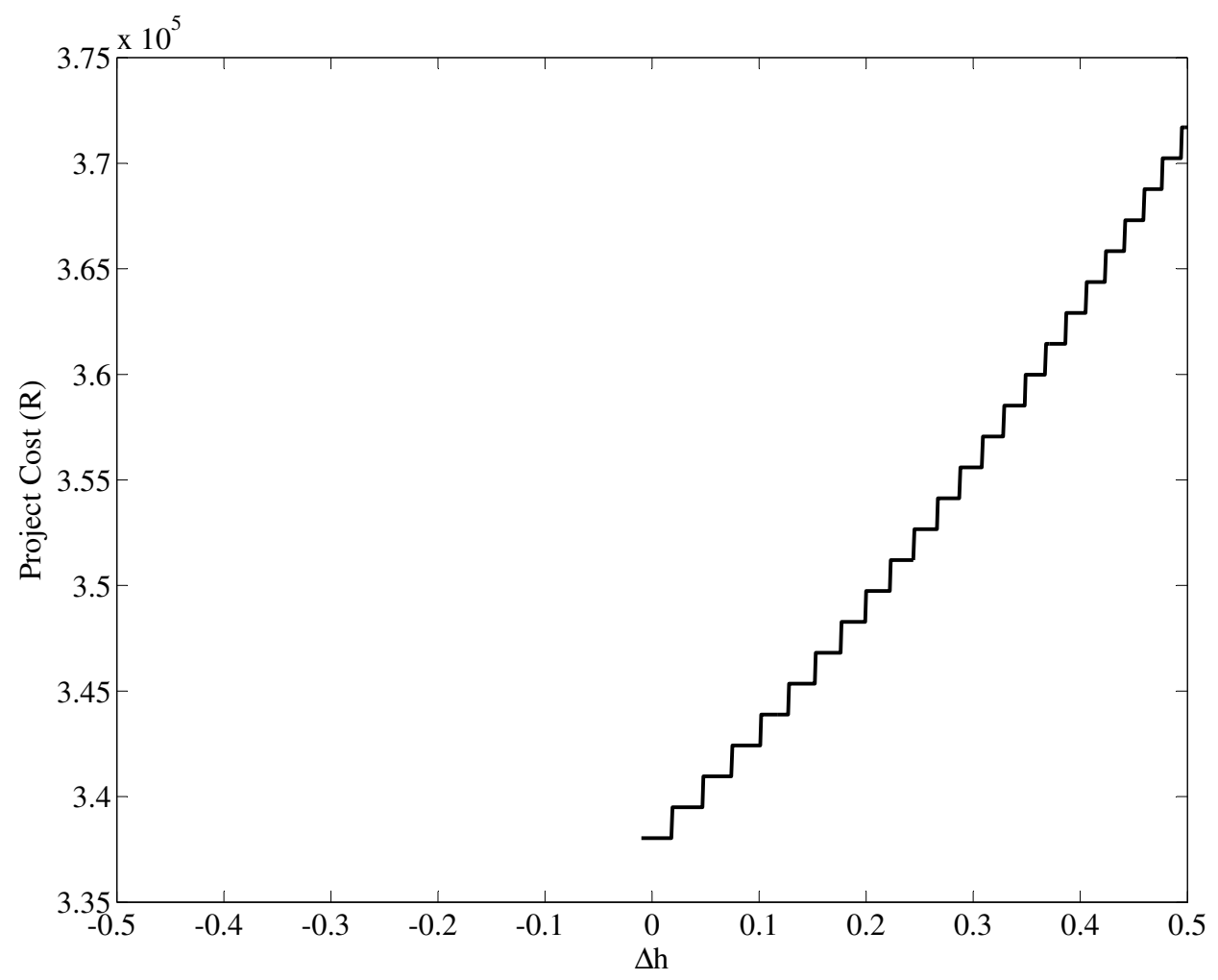

Figure 6: Cross section of solution at $\lambda_{7}+\Delta h$

was R339,942 (\$31,563) - 0.59\% higher than previous studies [13]. The sampling plan for this value is as follows:

$$
\mathbf{n}=(34,34,34,13,9,8,8,4,3,5,2) .
$$

It is also proposed that the reporting years be changed from $\delta=(2,4,6,8,10)$ to $\delta=(1,2,4,6,8,10)$. This is because one would expect the reported energy usage during the baseline phase (part of year 1) to adhere to the 90/10 criterion just as much as any other reporting year. In fact, if statistical power were a consideration, it would be more cost-effective to increase the baseline measurement accuracy, rather than to compensate on all subsequent accuracies. This may be a topic for future investigation. With this new constraint to year 1 as well as the baseline period (assumed to be taken together [13]), a solution is found to be:

$$
\mathbf{n}=(68,68,28,16,8,8,6,6,4,4,2),
$$

at a cost of R545,760 (\$50,674). It is noted that adhering to the $90 / 10$ criterion during the baseline phase adds significantly to project costs.

\begin{tabular}{cc} 
& Table 3: Optimisation Parameter Values \\
\hline Parameter name & Value \\
\hline Algorithm & GA \\
TolFun & $10^{-12}$ \\
TolCon & $10^{-12}$ \\
Population Size & 500 \\
$\lambda_{0}$ & $(1.645,0.1,1.645,0.1, \ldots)$ \\
\hline
\end{tabular}




\subsection{Model Comparison}

The first model improvement considered was that of the non-linear PELP population decay rate model proposed in equation (7). The PELP model predicts $8.3 \%$ more electricity savings than the CDM population decay rate model for the current case study parameters. The optimisation heuristic could not find a better solution to this problem than the one found for the CDM decay curve. The two sampling regimes could be different, though, with the CDM curve requiring smaller samples because of the finite population adjustment. More research is warranted in this area, but if the sampling plan proposed for a CDM model were used when in fact the PELP study is closer to the true decay shape, the 90/10 criterion would not be adhered to because the population difference would require a different finite population adjustment factor as incorporated into equation (36). The population would be undersampled, and savings underestimated.

The parameters of the PELP model were set to induce the same population at the end of the study than was present in the original study. As such, $\beta=0.543, \gamma=0.99$. The model converged to the following result:

$$
\mathbf{n}=(68,68,28,16,8,8,6,6,4,4,2),
$$

at a cost of $\mathrm{R} 545,760(\$ 50,674)$.

Second, and additionally, the exponential windowing function of equation (44) was introduced. As would be expected, since older and more recent results are not weighted equally, more samples would be required to adhere to the $90 / 10$ criterion. The result was

$$
\mathbf{n}=(68,68,33,16,28,21,19,16,16,15,20)
$$

at a cost of R696,552 $(\$ 64,675)$.

Last, the time-value of money was also taken into account. Since costs are now calculated in a different way, these results cannot be compared to previous results. A sampling regime for this consideration is:

$$
\mathbf{n}=(68,68,32,31,20,16,18,20,16,14,20)
$$

at a cost of $\mathrm{R} 1,459,121(\$ 135,480)$.

The cost of equation (47) in terms of the economic model is R1,199,061 showing that the proposed changes increase monitoring costs by $21,4 \%$ in NPV terms. This may be taken to mean that earlier models underestimated true project costs by this amount.

A comparison of the three proposed modifications (excluding the improvement on the previous optimal solution equation (46) or the correction of the baseline reporting requirement equation (47)) is plotted in Figure 7.

\subsection{Discussion of Results}

As expected, number of meters never exceed 68 in any given year, as indicated by equation (34). However, each subsequent model improvement considered does increase the cost of metering above the previous case.

Depending on the size of the population, possible increased metering cost due to using the sigmoid decay curve rather than the CDM straight-line curve may be offset by the increase in true savings reported. In the present study, the sampling plans are identical.

The second improvement - the exponential windowing function - does not allow the model to rely on high initial sample sizes to support much lower sample sizes in later years. The smoothing parameter was set at $\epsilon=0.25$ (a time constant of 4), although it should be case-dependent and determined by the $\mathrm{M} \& \mathrm{~V}$ engineer's judgement.

The last improvement, taking the time-value of money into account, also tends to shift metering towards the end of the study. This is because meter cost was modelled as depreciating, reinforced by opportunity costs which also penalise early expenditure on 'expensive' meters, when money could be invested and spent later on 'less expensive' meters. It is interesting to note that introducing this consideration does not increase sample sizes significantly, although it does skew their size distribution towards the later years of the study. The increased cost of this consideration in NPV terms is $0.5 \%$ when compared to the exponential windowing function modification. However, if $\varepsilon$ were changed to a different value, this comparison may well show a greater discrepancy.

Although this model may be applied to technologies other than lighting, caution should be exercised in such cases. This model is derived from first principles, but certain assumptions have been made that need to be adhered 


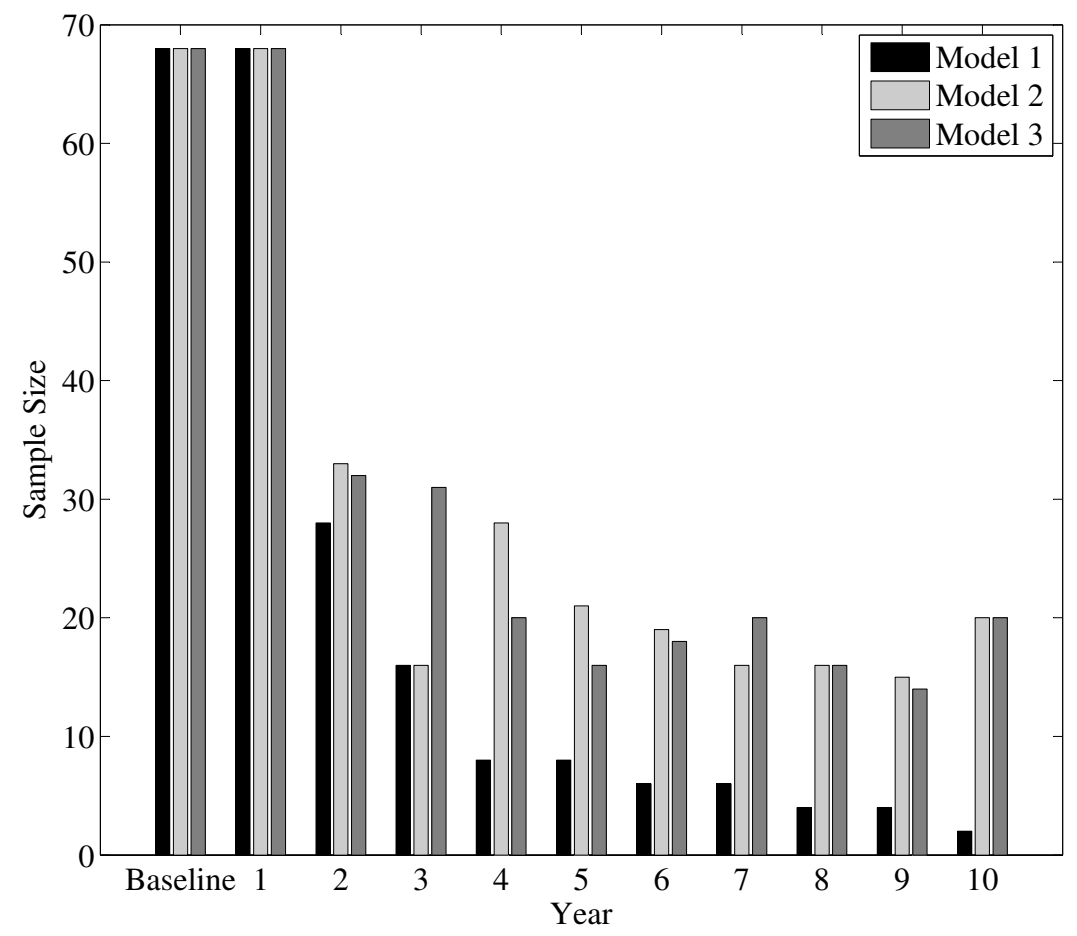

Figure 7: Comparison of three proposed model modifications. Model 1: equation (48), Model 2: equation (49), Model 3: equation (50)

to ensure compliance to the $90 / 10$ reporting criterion. First, lighting retrofit projects usually have large population sizes, making them suitable for statistical analysis. Second, normally distributed residuals are also assumed; this may not always the case for other technologies, and the engineer involved should consider this possibility. Third, lighting technologies are relatively insensitive to seasonality effects such as outside air temperature. For heating technologies where such covariates may be significant, it is recommended that an optimal sampling model be derived from the ASHRAE models [6] described in Section 2.1. Simple power meters may therefore not be suitable for such studies, and a combined uncertainty analysis is then warranted. Last, a CV value is assumed at the commencement of the study, but it may be underestimated as a small CV leads to smaller sample sizes and lower costs. However, sampling done on such a basis results in inadequate confidence or precision during reporting. Therefore it should be tested over a short period of time, and then updated, bearing in mind that seasonal effects may affect this value. In long-term projects the $\mathrm{CV}$ value should be evaluated on an annual basis.

\section{Conclusion and Future Work}

A number of improvements to current sampling design studies have been proposed and implemented, altering the sampling regime and cost significantly.

The improved CFL population survival model was found to fit known data very well, with a mean squared error (MSE) of 0.0015 compared to an MSE of 0.0368 for the previous model. The new model in discretised form also allows for optimal control theory to be applied to the problem with $P_{k+1}=f\left(P_{k}, u_{k}\right)$, and also reports greater savings than existing linear models.

By plotting line sections through the search space and investigating the gradient around known solutions, the nature of the search space was proven to be stepped and discontinuous, and the Genetic Algorithm was determined to 
be an appropriate heuristic for optimisation. The model was validated by applying it to certain test cases and checking performance against test cases. This approach allows for greater confidence in optimisation results.

A more accurate cumulative sampling function was devised and implemented. This function allows for the exponential decay of weights on past data, thereby increasing the relative contribution of more recent data during mean calculation.

An economic model incorporating the time-value of money was also implemented. This model not only accounts for inflation, but also takes investment opportunity costs and the difference between labour and capital costs into account. Using a minimum acceptable rate of return of $10 \%$ and an interest rate of $6.44 \%$ based on Consumer Price inflation, it was found that the project costs for optimal sampling plans are $21.4 \%$ higher in Nett Present Value (NPV) terms than previously calculated, although this figure is dependent on project-specific assumptions.

Neither the improved population survival model nor the addition of NPV considerations altered the sampling plans in a notable way, contributing $0 \%$ and $0.5 \%$ respectively. However, not weighting all samples equally did have a significant effect, contributing the bulk of the increased project cost.

\subsection{Recommendations}

Although numerous improvements to the aforementioned changes may be made, it is recommended that future work focus on the sensitivity of the model to the latent assumptions identified in existing literature on longitudinal CFL sampling design: that the mean energy usage is stationary throughout the study, which limits the resolution of surveillance to annual calculation, and that samples taken in different years are independent. The statistical power during sample size calculation should also be considered, as well as the structure of meter pricing and contracting schemes. A future study may investigate the sensitivity of the model to these assumptions.

\section{Acknowledgement}

This work is supported by the Centre for New Energy Systems and the National Hub for the Postgraduate Programme in Energy Efficiency and Demand Side Management at the University of Pretoria.

[1] Eskom, Eskom Integrated Report 2011, Electronic Publication (2011).

URL http://financialresults.co.za2011eskom_ar2011.

[2] Eskom, The Measurement and Verification Guideline for Energy Efficiency and Demand-Side Management (EEDSM) Projects and Programmes, Eskom Corporate Services Division, Assurance and Forensic Department, 10th Edition (January 2010).

[3] UNFCCC, Approved Small-Scale Methodology AMS II.J, Demand-Side Activities for Efficient Lighting Technologies (May 2013).

[4] EVO, International Performance Measurement and Verification Protocol Vol. 1, Efficiency Valuation Organization, EVO 10000 - 1:2012 Edition (January 2012).

[5] ISO New England, Manual for Measurement and Verification of Demand Reduction Value from Demand Resources (M-MVDR), 2nd Edition (2010).

[6] American Society of Heating, Refrigeration and Air-Conditioning Engineers, Inc., ASHRAE Guideline, Measurement of Energy and Demand Savings, 14th Edition (June 2002). URL http: //www . ashrae.org

[7] S. Schiller, C. Goldman, E. Galawish, National energy efficiency evaluation, measurement and verification (em\&v) standard: Scoping study of issues and implementation requirements, Tech. rep., U.S. Department of Energy Office of Electricity Delivery and Energy Reliability (April 2011).

[8] X. Xia, J. Zhang, Mathematical description for the measurement and verification of energy efficiency improvement, Applied Energy 111 (0) (2013) 247 - 256. doi:http://dx.doi.org/10.1016/j.apenergy.2013.04.063.

[9] T. Reddy, D. Claridge, Uncertainty of measured energy savings from statistical baseline models, HVAC\&R Research 6 (1) (2000) 3-20.

[10] CDM Executive Board, Approved baseline and monitoring methodology am0046, Tech. rep., UNFCCC (2007).

[11] A. Michaelowa, D. Hayashi, M. Marr, Challenges for energy efficiency improvement under the CDM - the case of energy-efficient lighting, Energy Efficiency 2 (4) (2009) 353-367.

[12] X. Ye, X. Xia, J. Zhang, Optimal sampling plan for clean development mechanism energy efficiency lighting projects, Applied Energy 112 (2013) 1006-1015.

[13] X. Ye, X. Xia, J. Zhang, Optimal sampling plan for clean development mechanism lighting projects with lamp population decay, submitted to Applied Energy (August 2013).

[14] G. E. Box, G. M. Jenkins, G. C. Reinsel, Time Series Analysis: Forecasting and Control, John Wiley \& Sons, 2013.

[15] D. Ruch, J. Kissock, T. Reddy, Prediction uncertainty of linear building energy use models with autocorrelated residuals, Journal of Solar Energy Engineering 121 (1) (1999) 63-68.

[16] J. Neter, W. Wasserman, M. Kutner, Applied linear regression analysis, Homewood, IL: Irwin.

[17] P. O'Connor, A. Kleyner, Practical Reliability Engineering, Wiley, 2011.

[18] The TecMarket Works Team, California energy efficiency evaluation protocols: Technical, methodological, and reporting requirements for evaluation professionals (April 2006). 
[19] Navigant Consulting, Evaluation of the IFC/GEF Poland Efficient Lighting Project CFL Subsidy Program, Tech. Rep. 1, Netherlands Energy Efficient Lighting B.V., International Finance Corporation / Global Environment Facility (1999).

[20] Resource Management International, Inc (RMI), Rockland electric residential lighting persistence study, fourth quarter, 1998 results, Tech. rep., Prepared for Orange and Rockland Utilities, Bala Cynwyd, Pennsylvania (November 1998).

[21] M. Botha-Moorlach, G. Mckuur, A report on the factors that influence the demand and energy savings for compact fluorescent lamp door-todoor rollouts in south africa, Tech. rep., Eskom (March 2009).

[22] P.-F. Verhulst, Notice sur la loi que la population poursuit dans son accroissement, Correspondance mathematique et physique 10 (1838) $113-121$.

[23] A. J. Lotka, Elements of Physical Biology, Williams \& Wilkins Company, Balitmore, 1925.

[24] H. Carstens, X. Xia, X. Ye, J. Zhang, Characterising compact fluorescent lamp population decay, in: IEEE Africon Conference, Pointe-AuxPiments, Mauritius, 2013

[25] CDM Executive Board, Standard for sampling and surveys for cdm project activities and programme of activities, Tech. rep., UNFCCC (2012).

[26] A. Baker, Sample size selection in energy efficiency research and evaluation the use and abuse of the coefficient of variation, in: International Energy Program Evaluation Conference, Research Into Action, Rome, Italy, 2012.

[27] W. A. Fuller, Sampling Statistics, John Wiley \& Sons, 2011.

[28] South African Reserve Bank, Statistical Release P0141: Consumer Price Index (August 2013).

[29] CDM Executive Board, Project design document: Gauteng, free state, mpumalanga, limpopo \& northern cape cfl replacement project in south africa, Tech. rep., UNFCCC (2006). 